\title{
Biophysical profiling of tumor cell lines
}

\author{
Frederick Coffman ${ }^{\mathrm{a}, *}$, Rachid Hamid ${ }^{\mathrm{b}}$, Marion C. Cohen ${ }^{\mathrm{c}}$, Ralph Garippa ${ }^{\mathrm{b}}$ and Stanley Cohen ${ }^{\mathrm{a}}$ \\ ${ }^{a}$ Department of Pathology, UMDNJ - New Jersey Medical School, Newark, NJ, USA \\ ${ }^{\mathrm{b}}$ Roche Discovery, Roche Scientific, Nutley, NJ, USA \\ ${ }^{\mathrm{c}}$ Department of Pathology, SUNY Downstate Medical Center, Brooklyn, NY, USA
}

\begin{abstract}
Despite significant differences in genetic profiles, cancer cells share common phenotypic properties, including membrane-associated changes that facilitate invasion and metastasis. The Corning Epic ${ }^{\circledR}$ optical biosensor was used to monitor dynamic mass rearrangements within and proximal to the cell membrane in tumor cell lines derived from cancers of the colon, bone, cervix, lung and breast. Data was collected in real time and required no exogenously added signaling moiety (signal-free technology). Cell lines displayed unique profiles over the time-courses: the time-courses all displayed initial signal increases to maximal values, but the rate of increase to those maxima and the value of those maxima were distinct for each cell line. The rate of decline following the maxima also differed among cell lines. There were correlations between the signal maxima and the observed metastatic behavior of the cells in xenograft experiments; for most cell types the cells that were more highly metastatic in mice had lower time-course maxima values, however the reverse was seen in breast cancer cells. The unique profiles of these cell lines and the correlation of at least one profile characteristic with metastatic behavior demonstrate the potential utility of biophysical tumor cell profiling in the study of cancer biology.
\end{abstract}

Keywords: biophysical measurement, mass redistribution, Epic ${ }^{\circledR}$ optical biosensor, tumor cells, metastasis

\section{Background}

Oncogenesis involves the accumulation of mutations in many genes that results in the dysregulation of numerous interrelated pathways. The pattern of cancerrelated mutations differs from one type of cancer to another, and even within single types of cancer, multiple gene expression patterns have been catalogued [26-28]. Efforts to elucidate gene expression profile fingerprints predictive of cancer clinical behavior are ongoing but have been hampered in no small part due to this variability [16].

Despite the variable expression patterns at the molecular level, tumor cells share several common phenotypes, including genetic instability, dysregulated control of proliferation, defects in apoptosis, and invasive and metastatic capabilities. The acquisition of invasive and metastatic capabilities represents a critical

${ }^{*}$ Corresponding author: Frederick Coffman, Department of Pathology, UMDNJ - New Jersey Medical School, 185 S. Orange Avenue, Newark, NJ 07103, USA. E-mail: coffmafd@umdnj.edu. clinical landmark, as over $90 \%$ of cancer patient mortality is due to metastases [21, 22]. The processes of invasion and metastasis require activities within or proximal to the cell membrane, including interactions with extracellular matrix components and with cells comprising the vascular system, the lymphatic system, and tissues where metastatic cells establish secondary tumor masses [21].

The metastasis-linked changes in cellular membrane activities, caused by oncogenesis-linked changes in gene expression, unavoidably alter the biophysical properties of cells. Instruments exist which are capable of measuring cell biophysical properties, including surface plasmon resonance, optical biosensors, and impedance biosensors, but they have been primarily utilized for high throughput screening of bioactive agents, such as agonists and antagonists of $\mathrm{G}$ protein coupled receptors $[7,20]$. We hypothesize that because transformed cells exhibit commonly shared phenotypic features, the biophysical profiles of transformed cells, both intrinsic and in response to external stimuli, contain commonalities as well. We further hypothesize that 
these biophysical profiles are reflective of basic biological properties of these cancer cells and in some cases may be predictive of the cancers' clinical behavior.

We here utilize the Corning Epic ${ }^{\circledR}$ system, which contains refractive waveguide grating optical biosensors that measure changes in cellular refractive indexes within and proximal to the cell membrane, within a detection zone extending approximately $150 \mathrm{~nm}$ into the cell from the cell membrane via surface plasmon resonance-related phenomena $[6,18]$. Changes in refractive indices are due to dynamic mass rearrangements within the detection zone; among the activities that can contribute to this signal are receptor activation, receptor dimerization, receptor internalization, cytoskeleton rearrangements, changes in cell adhesion, changes in cell shape, and endo- and exocytosis. Data was collected in real time on cell lines derived from a number of different cancers. Our results indicate that not only does each cell type have unique biophysical profiles, but certain characteristics of the response time-courses appear to correlate with the in vivo behavior of these cell lines as described in the literature.

\section{Materials and methods}

\subsection{Cells}

A549, U-2 OS, Saos 2, CHO-K1, HT 29, LS174T, Hela, T84, MCF12A, and MCF-7 were obtained from ATCC. Cells were grown at $37^{\circ} \mathrm{C}$ in an atmosphere of $5 \% \mathrm{CO} 2$, in the media recommended for each individual cell line: F-12K with $10 \%$ FBS (A549, U-2 OS, CHO-K1), McCoys 5A with 10\% FBS (Saos 2, HT29, HCT 116, LS174T), DMEM with $10 \%$ FBS (Hela, T84, MCF-7), and 1:1 DMEM: Ham's F12 with $10 \%$ FBS, $20 \mathrm{ng} / \mathrm{ml}$ EGF, $100 \mathrm{ng} / \mathrm{ml}$ cholera toxin, $10 \mu \mathrm{g} / \mathrm{ml}$ insulin, and $500 \mathrm{ng} / \mathrm{ml}$ hydrocortisone (MCF12A). LCC1 and LCC2 cells were a generous gift of Dr. Robert Clarke [2], and were grown in IMEM without phenol red supplemented with $10 \%$ FBS that had been charcoal treated to remove steroid hormones.

\subsection{Biophysical profiling}

Cells were harvested using trypsin in $1 \mathrm{mM}$ EDTA, washed, and seeded in 384 well Epic $^{\circledR}$ microplates at a density of $3.5 \times 10^{4}$ cells/well. The plates were placed into the unit and data collection was begun immediately or at a defined time after seeding. The microplate base contains a thin refractive grating that is illuminated by a broadband source, which is sensitive to changes in the index of refraction within a detection zone extending $150 \mathrm{~nm}$ into the cells. The changes in index of refraction are due to dynamic mass rearrangements within the detection zone, linked to molecular aggregation and dissociation, cytoskeletal rearrangements, lipid raft movements, and other changes in macromolecular structures [6]. Data points were collected every 45 seconds. Each data point is the average of 5 scans over different parts of an individual well.

\section{Results}

We began with a survey of tumor cell lines from diverse tissue origins, including cancers of the breast, bone, cervix, lung, and colon. Data from replicate wells are very reproducible as shown in Figs. 1 and 3B. All of the wells containing a particular cell line produced response values that were quite close at any given time point with identical curve shapes. As seen comparing Figs. 1-4, the time-courses produced by each individual cell line are qualitatively and quantitatively different in three areas. One is the time to reach a maximal value, which ranged from 30 minutes to three and a half hours. Two is the maximal value of each curve, which varied greatly from the lowest (LS174T) to the highest (Saos-2) values. Three is the slope of the curve diminishing with time from that maximal value. These parameters are affected by the time data collection is initiated relative to the time the plates were seeded. If there was a significant lag between the time the plates were seeded and the initiation of data collection, from 30 minutes to 12 hours (overnight), the shapes of the curves and the maximum values were affected, but each cell line maintained individual timecourse characteristics (Figs. 2 and 3). There does not appear to be a point within the first 12 hours where, if data collection is started at that point, all cell lines produce the same time-course profile. This indicates that the parameters creating the time-course characteristics are intrinsic properties of the individual cell lines.

Two of the three qualitative aspects of each timecourse mentioned above can be easily measured - the maximal value, and the time required to reach the maximal value. Experiments examining a number of different tumor cell types were performed in which data was collected immediately after cell seeding. A plot of the maximal values for each cell line and the 

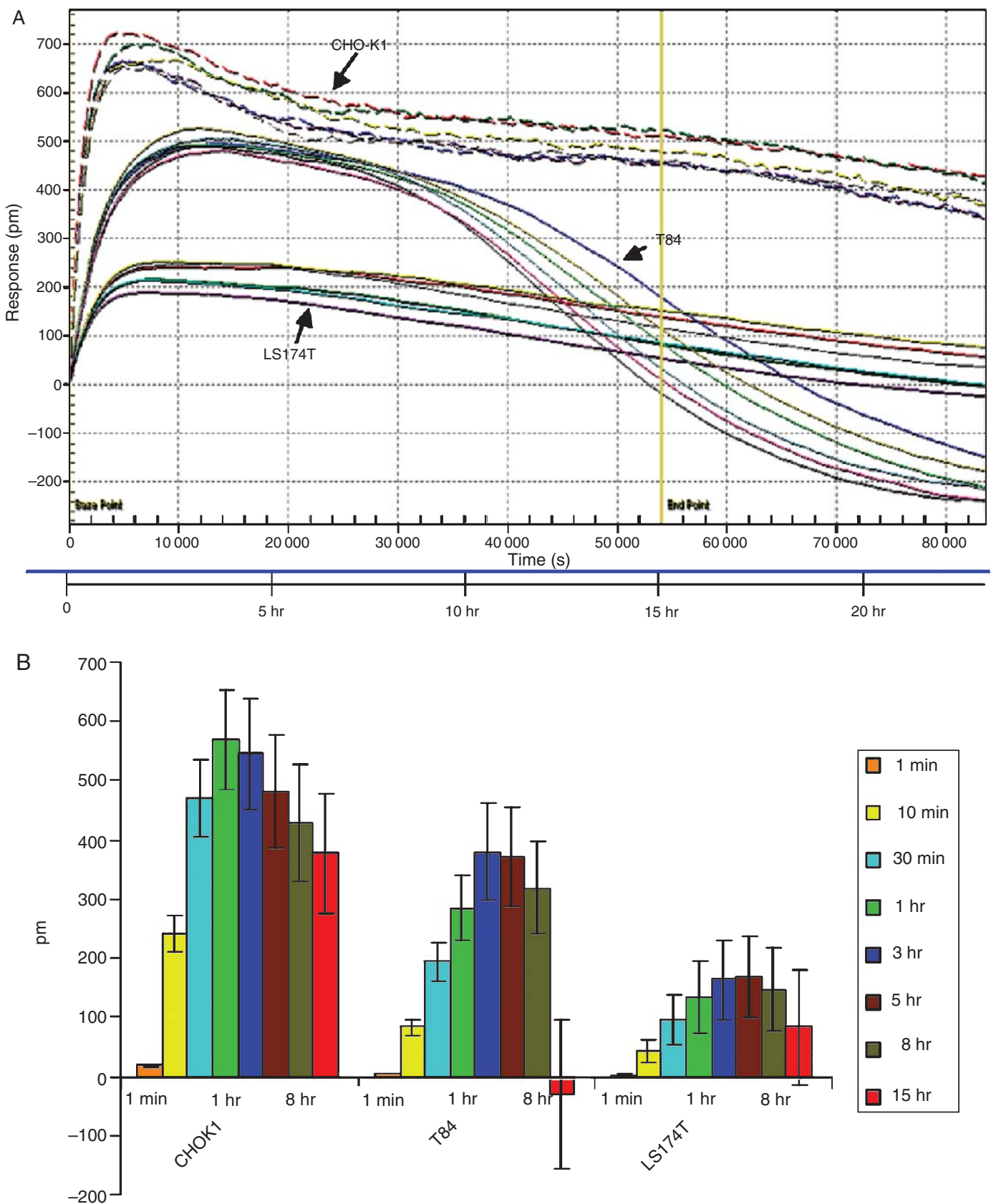

Fig. 1. Time-course profiles showing reproducibility of time points in replicate wells. A) Top curve: CHO-K1, middle curve T84, bottom curve LS174T. B) Histograms of values at defined time points.

time needed to attain the maximum values (Fig. 5) shows that within each tumor type, the cells that display a higher degree of metastatic behavior in mouse xenograft experiments (Table 1) generally have lower maxima values in their time-courses. There appears to be some differences among tumor cell types, for example the bone cancer cells as a group tend to have higher maxima values than the colon cancer cells, 


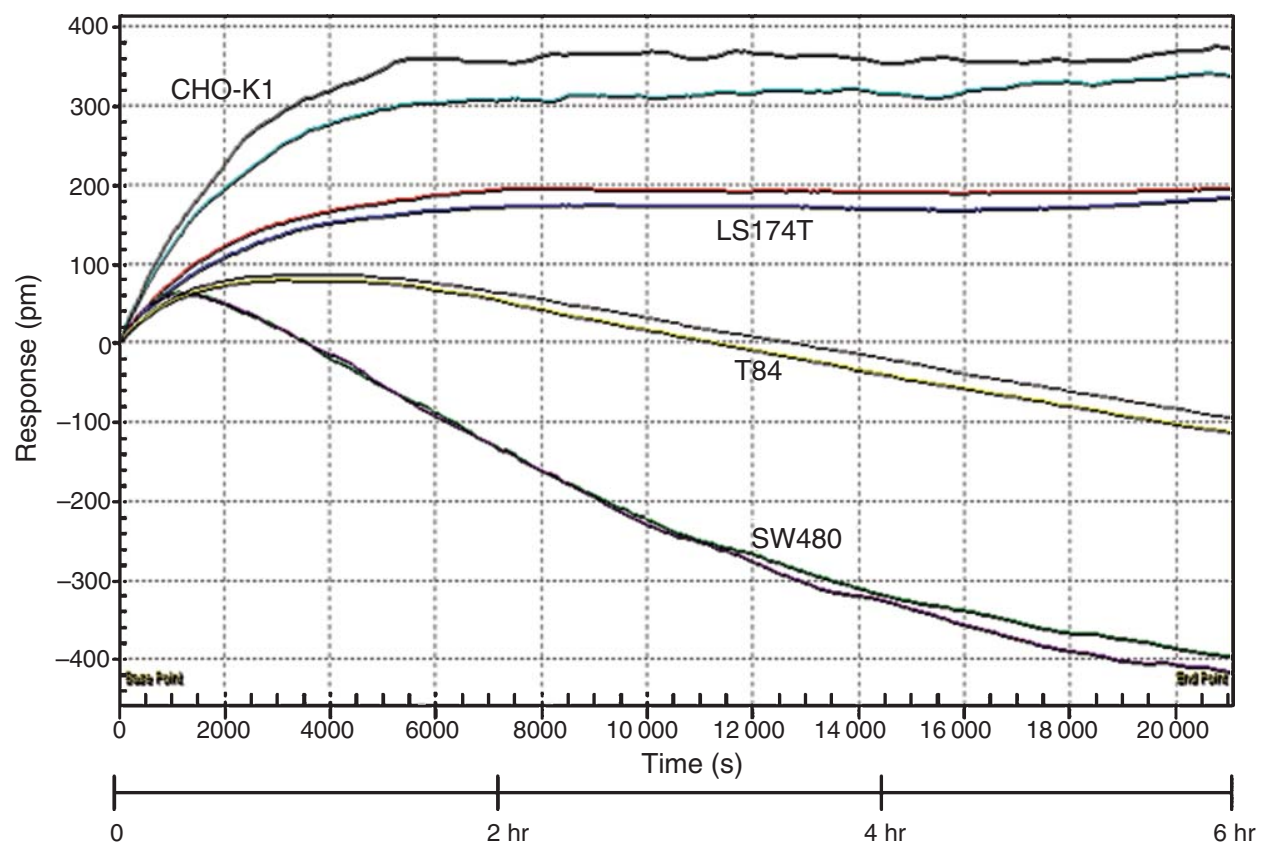

Fig. 2. Time-course profile of cell lines beginning 30 minutes after seeding. Top curve CHO-K1, second curve LS174T, third curve T84, bottom curve SW480.

which may reflect tissue type characteristics. Interestingly, the CHO-K1 cells, which are nontumor cells and produced relatively high maximam values, are weakly metastatic when introduced into immunocompromised mice. From the data collected to date, there does not appear to be a correlation between the time to reach the maximal value and the metastatic behavior of those cells in mouse xenograft model systems.

With this as background, we undertook a more direct and systematic comparison of several breast cancer cell lines of known increasing metastatic potential. MCF-12A cells are a normal breast epithelial cell line. MCF-7 is a breast adenocarcinoma cell line that is locally invasive but not metastatic, and the LCC1 and LCC2 cell lines are metastatic variants of MCF-7 cells [2]. As shown in Fig. 6, the nontumorigenic MCF-12A cells have a significantly higher signal maxima compared to the three breast cancer lines. However, the nonmetastatic but locally invasive MCF7 cells produce lower signal maxima than the metastatic LCC1 and LCC 2 cells, which is the reverse of the behavior seen in other tumor cell types. The LCC 1 and LCC 2 cells have nearly identical maximum values and for both cell lines the time required to reach this maximum is the same, however the slopes of the time-course following the maxima differ (LCC1 cell signals decline significantly faster than LCC2 signals). That the general observation that the more metastatic cells generally have lower maxima was not followed in this set of breast cancer cells suggests that these complex signals may be affected differentially in different tumor cell types.

\section{Discussion}

Countless studies have documented changes in gene expression patterns in tumor cells, which are complex and variable among different tumors and even among tumors of the same type. While changes in the expression patterns of a limited number of genes have been correlated with oncogenic progression, and in some cases, such as in triple negative breast cancer, have clinical relevance [26], more widely useful gene expression fingerprints remain to be elucidated. However, the shared phenotypes of cancer cells, including invasive and metastatic capabilities of advanced stage tumors, suggest that numerous gene 

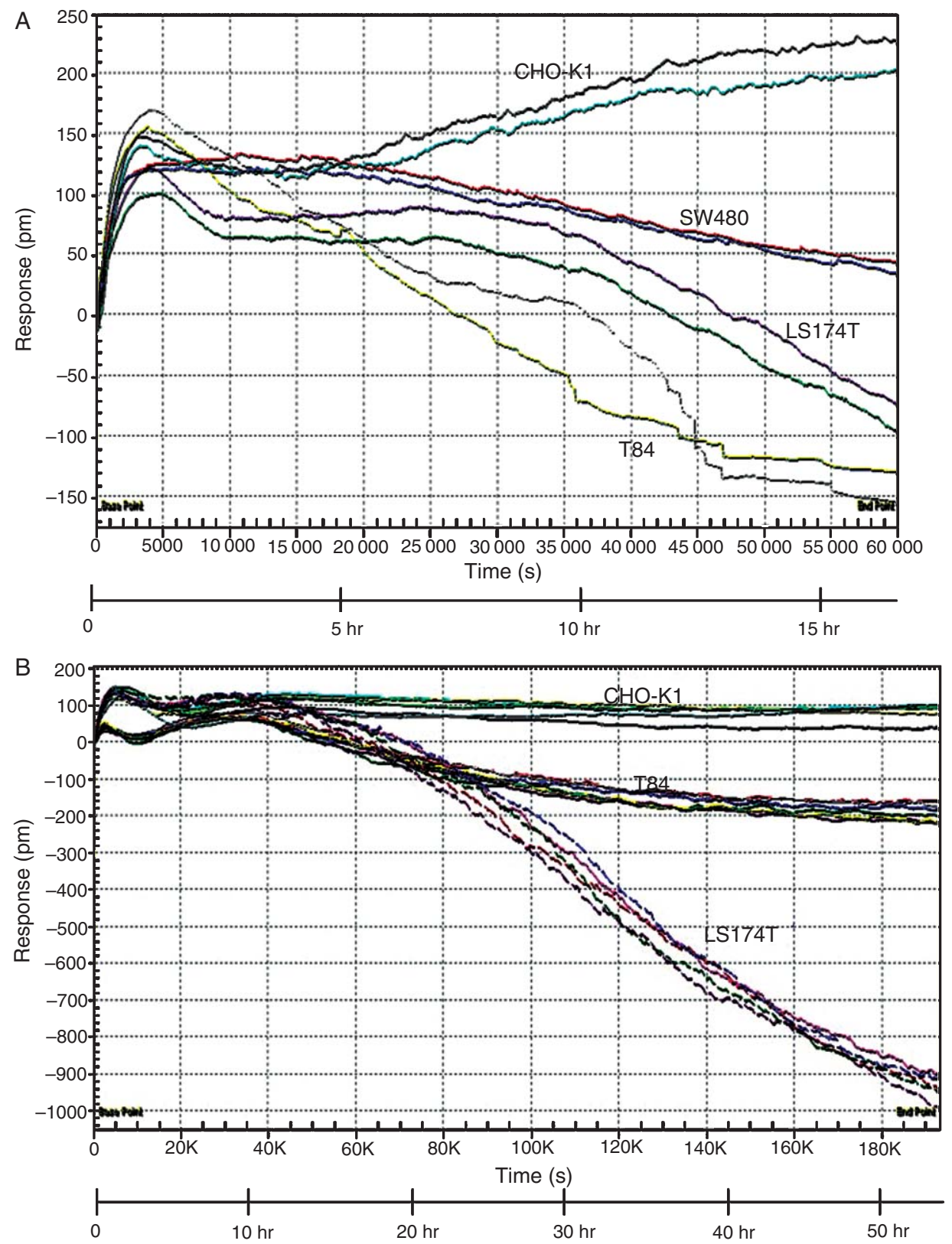

Fig. 3. A) Time-course profiles of four cell lines following overnight attachment and incubation at $37^{\circ} \mathrm{C}$. Top curve CHO-K1, second curve SW480, third curve LS174T, bottom curve T84. B) Long term profile of three cell lines following overnight attachment. Top curve CHO-K1, middle curve T84, bottom curve LS174T.

expression changes can result in a limited number of common phenotypic changes. These studies represent part of an initial exploration of tumor cell biophysical properties that would be relevant to inva- sion and metastasis, as the $150 \mathrm{~nm}$ detection zone of the Epic ${ }^{\circledR}$ instrument contains molecules involved in tumor cell migration, cell matrix interactions, and interactions with tissue cells. The changes in mass 


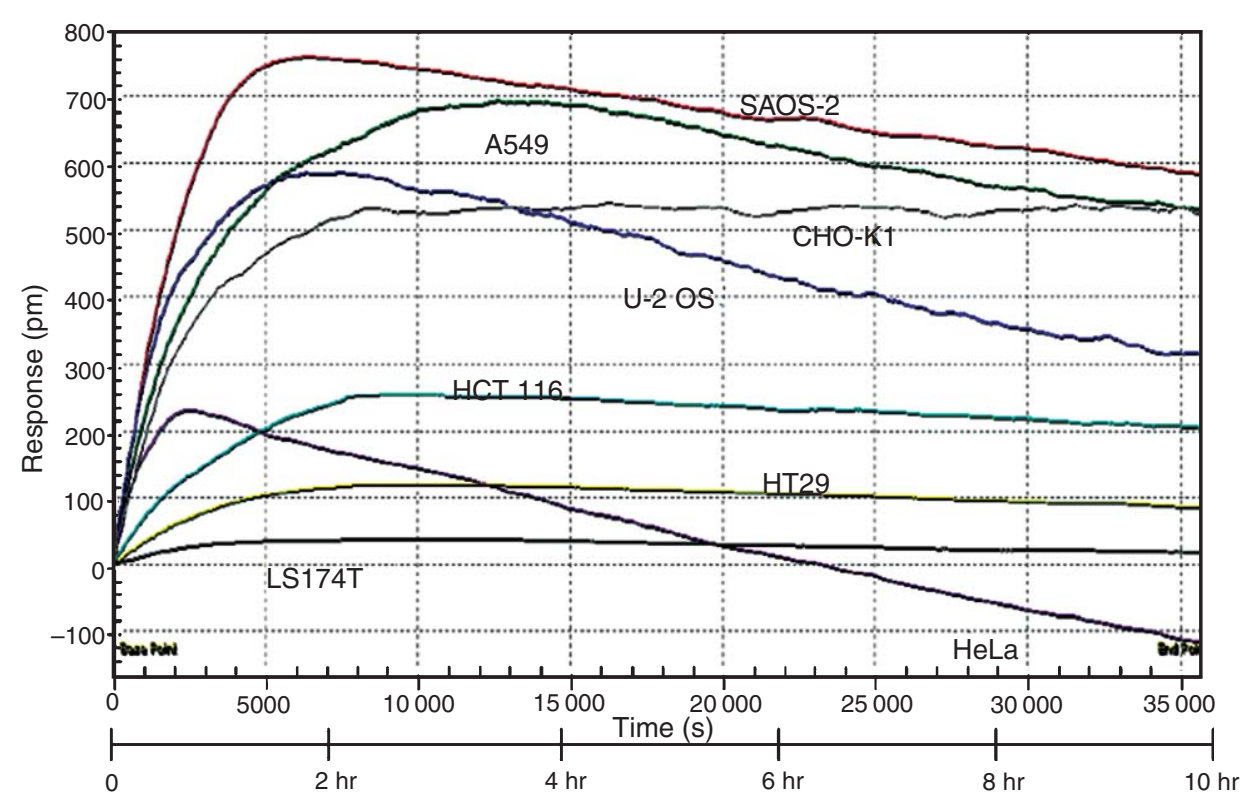

Fig. 4. Comparative time-course profiles of multiple tumor type cell lines. Top (red) curve SAOS-2, green curve A549, black curve CHO-K1, dark blue curve U-2 OS, light blue curve HCT 116, yellow curve HT29, grey curve LS174T, purple curve HeLa.

distribution measured by the Epic instrument represents a complex multicomponent signal, including cell growth and division, cell membrane composition, lipid rafts, membrane proteins, cytoskeletal elements, and other macromolecules within the $150 \mathrm{~nm}$ detection zone. Our initial concern that these signals would be dominated by factors relating to differential cell growth was alleviated by the lack of correlation of these signals with the published and observed proliferative rates of these cells. As differential rates of growth may impact future mechanistic studies, mechanistic studies will need to include cumulative cell counts. However, identifying individual phenomena that contribute to the time-courses may be less important than relating time-course behavior to tumor properties; indeed, the fact that the readout represents an integrative measure such as this avoids the noise of detailed and redundant molecular pathway data.

The relationship between the maximal value of the time-courses and the metastatic activities of the various cells in mouse xenograft experiments is difficult to quantify exactly due to the variability in methodologies among xenograft tumor models. Different xenograft studies used different numbers of cells to inoculate

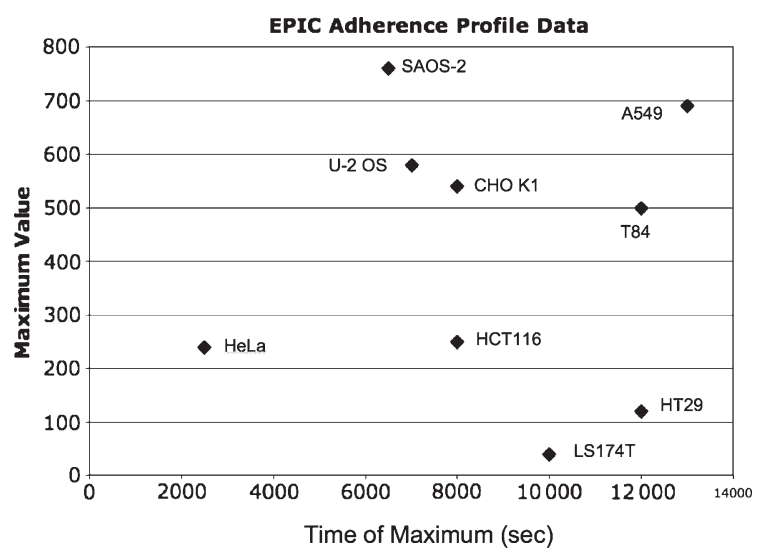

Fig. 5. Plot of maximal value of time-courses vs. time to achieve maximal value (data taken from Fig. 4).

the mice, the tumor cells are administered in different ways, the development of metastases are reported at different time points, and metastases are reported using different measures, all of which made direct comparisons difficult (Table 1). In addition, different tumor cell types tended to have different ranges of maximal values on the time-courses: bone cancer cells as 
Table 1

Metastatic behavior of human cancer cell lines in immunocompromised murine models

\begin{tabular}{lll}
\hline Cell Line & \multicolumn{1}{c}{ Tumor Type } & \multicolumn{1}{c}{ Metastasis } \\
\hline SAOS-2 & bone & $1 \times 10^{7}$ cells sc; tumors form over 30 weeks, no metastases [15] \\
& & $1 \times 10^{7}$ cells sc; tumors form over 4 weeks, 27\% of mice had pulmonary metastases in 12 weeks [29] \\
U-2 OS & bone & $2.5 \times 10^{6}$ cells i.v; $16 / 19(84 \%)$ showed lung metastases at 2 months [11] \\
HCT116 & colon & $5 \times 10^{6}$ cells injected subcutane only 6-8 -weeks 15/32 liver metastases, 13/32 liver metastases [17] \\
& & $5 \times 10^{6}$ cells injected into the spleen; 11/14 liver metastases [12] \\
HT29 & colon & $1 \times 10^{8}$ cells sc, in 30-35 days 481 metastases/30 mice [10] \\
LS174T & colon & $1 \times 10^{7}$ cells injected into the spleen, all developed liver metastases within 35 days [8] \\
& & $2 \times 10^{6}$ cells sc also developed liver metastases \\
T84 & colon & $2 \times 10^{6}$ cells tail vein injection, 4-5 weeks 42\% developed lung metastases [1] \\
HeLa & cervix & $1 \times 10^{5}$ cells in tail vein; in 30 daya all mice had lung metastases [24] \\
A549 & lung & $5 \times 10^{6}$ cells sc, all mice developed lung metaatasea [3] \\
CHOK1 & nontumor (ovary) & $1 \times 10^{6}$ cells s.c, tumors developed over 7-8 weeks, 20\% of mice also developed metastases [22] \\
& & $3 \times 10^{5}$ GFP-labeled cells iv micrometastases seen within 4 days [14] \\
MCF12A & nontumor (breast) & $2 \times 10^{6}$ cells injected into fat pad, no tumors or metastases seen at 12 weeks [5] \\
MCF7 & breast & $2 \times 10^{6}$ cells injected into fat pad, primary tumors but no metastases seen at 12 weeks [4, 25] \\
LCC1 & breast & $2 \times 10^{6}$ cells injected into fat pad, primary tumors and metastasis seen at 12 weeks [4, 25] \\
LCC2 & breast & $2 \times 10^{6}$ cells injected into fat pad, primary tumors and metatastasis seen at 12 weeks [4] \\
\hline
\end{tabular}

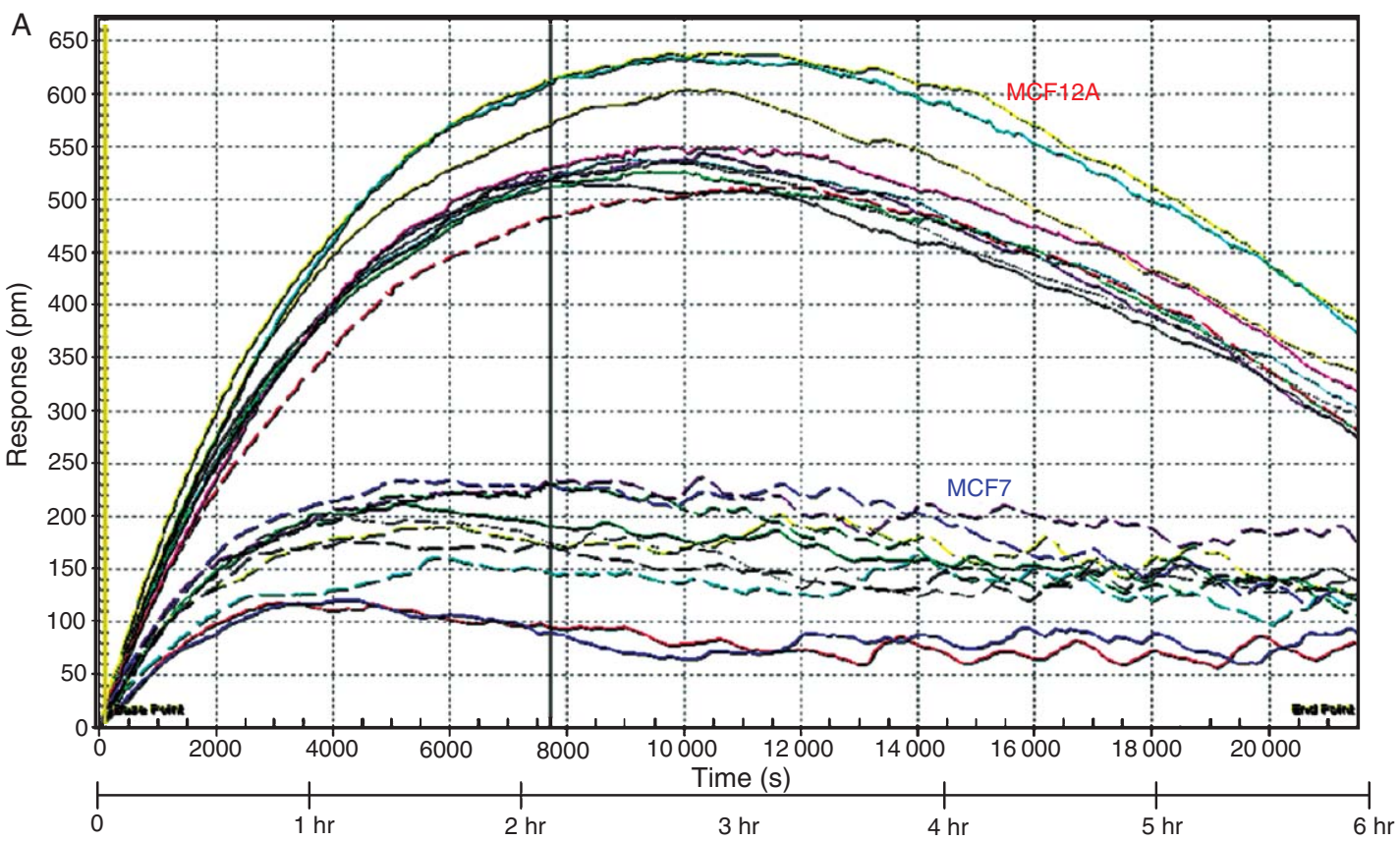

Fig. 6. Time-course comparison of breast cancer cell lines of increasing metastatic capability. A) Normal breast cell line MCF12A and nonmetastatic breast cancer cell line MCF7. B) Normal MCF12A, nonmetastatic MCF7, and metastatic LCC1 cell lines. C) Normal MCF12A, nonmetastatic MCF7, and metastatic LCC2. Fig. 1.

a group tended to have higher maximal values while colon cancer cells as a group tended to have lower values. However, except for the breast cancer lines, within each group, the more metastatic cells tended to have higher maxima values. CHO-K1 cells, a nontumor cell with a relatively high maxima value, is weakly metastatic in xenograft mouse models, while HeLa cells, a highly metastatic cervical carcinoma cell line, 

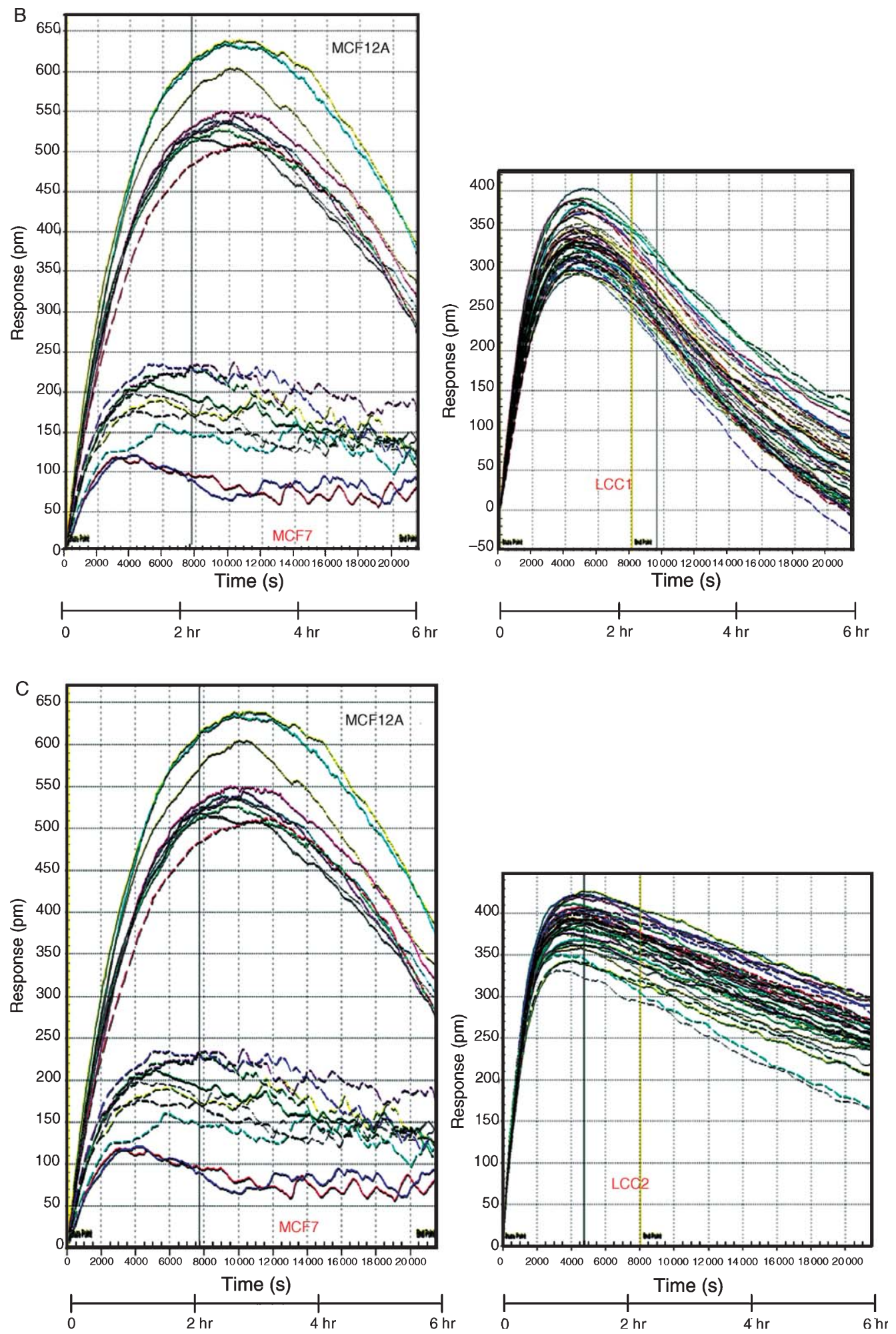

Fig. 6. Continued 
has a low maxima value. The other two parameters of the time-courses, the time required to reach the maximal value and the slope of the curve moving away from the maximal value, as yet show no correlation with the metastatic capabilities of the cells.

The Epic ${ }^{\circledR}$ instrument measures changes in the index of refraction (dynamic mass rearrangements) in a $150 \mathrm{~nm}$ detection zone which includes the cell membrane. Oncogenic changes in cell membranes, an important component of invasive and metastatic activities, have been reported previously. Changes in several membrane physical properties, including changes in protein and lipid composition and an accompanying increase in membrane fluidity, have been seen when comparing metastatic to nonmetastatic LM cells [9]. It has long been known that the cell surface carbohydrate composition of tumor cells differs from normal cells; increased sialic acid content being one difference [13]. Tumor cells have been seen to over express a number of surface proteins, some of which have clinical utility [28]. The substantial increase in a particular membrane protein will undoubtedly change the biophysical properties of the tumor cell. The relationship between tumor cell membrane changes and changes in dynamic mass redistribution is currently unclear, but represents a potentially fruitful avenue of experimentation.

The local microenvironment also plays a role in the metastatic activities of tumor cells. The coexistence of a tumor cell, a macrophage, and an endothelial cell has been shown to potentiate tumor cell intravasation in rodent carcinoma models, and the density of this tripartite arrangement, termed tumor microenvironment of metastasis (TMEM), was also shown to correlate with the development of systemic metastasis in breast cancer patients [19]. Thus optimal understanding of tumor cell biophysical behavior will likely require information about how tumor biophysical properties are affected by the surrounding cells.

While the short-term goal of these studies is to develop an understanding of the biophysical natures of different tumor cell types at a basic science level, the desired long term goal is to elucidate patterns within the biophysical profiles that can either directly or indirectly contribute to improved clinical outcomes. The present study involving cell lines demonstrates the validity of this concept and approach in achieving the short-term goal. To create more complete profiles, biophysical data will be correlated with gene expression data as well as other relevant observations, such as resistance to specific chemotherapeutic agents. In addition, it is unlikely that a single biophysical measurement will provide sufficient information for every tumor type. Viable tumor biophysical profiles will only emerge from the use of multiple complementary approaches, both to cope with innate differences in cell structure and composition, and to minimize the effects of technical artifacts. To that end, we are currently performing parallel time-course studies measuring impedance in these cell lines. The results we have obtained to date support the results reported here. These studies support our contention that utilizing multiple approaches to create more complete biophysical profiles will provide relevant information about tumor cells.

\section{References}

[1] R. Alessandro, A.M. Flugy, D. Russo, G. Stassi, A. De Leo, C. Corrado, G. Alaimo and G. De Leo, Identification and phenotypic characterization of a subpopulation of T84 human colon cancer cells after selection on activated endothelial cells, J Cell Physiol 203 (2005), 261-272.

[2] N. Brunner, T.L. Frandsen, C. Holst-Hansen, M. Bei, E.W. Thompson, A.E. Wakeling, M.E. Lippman and R.E. Clarke, MCF7/LCC2: A 4-hydroxytamoxifen resistant human breast cancer variant that retains sensitivity to the steroid antiestrogen ICI 182, 780, Cancer Res 53 (1993), 3229-3232.

[3] K.X. Cai, L.Y. Tse, C. Leung, P.K.H. Tam, R. Xu and M.H. Sham, Suppression of lung tumor growth and metastasis in mice by adeno-associated virus-mediated expression of vasostatin, Clin Cancer Res 14 (2008), 939-949.

[4] T.A. Castro, M.C. Cohen and P. Rameshwar, The expression of neurokinin-1 and preprotachykinin-1 in breast cancer cells depends on the relative degree of invasive and metastatic potential, Clin Exp Metastasis 22 (2005), 621-628.

[5] R.D. Coletta, K.L. Christensen, D.S. Micalizzi, P. Jedlicka, M. Varella-Garcia and H.L. Ford, Six 1 overexpression in mammary cells induces genomic instability and is sufficient for malignant transformation, Cancer Res 68 (2008), 2204-2213.

[6] H.N. Daghestani and B.W. Day, Theory and applications of surface plasmon resonance, resonant mirror, resonant waveguide grating, and dual polarization interferometry biosensors, Sensors 10 (2010), 9630-9646.

[7] Y. Fang and A.M. Ferrie, Label-free optical biosensor for ligand-directed functional selectivity acting on 32 adrenoceptor in living cells, FEBS Lett 582 (2008), 558-564.

[8] T.L. Kalber, J.C. Waterton, J.R. Griffiths, A.J. Ryan and S.P. Robinson, Longitudinal in vivo susceptibility contrast MRI measurements of LS174T colorectal liver metastasis in nude mice, J Mag Res Imaging 28 (2008), 1451-1458.

[9] A.B. Kier, Membrane properties of metastatic and nonmetastatic cells cultured from $\mathrm{C} 3 \mathrm{H}$ mice injected with $\mathrm{LM}$ fibroblasts, Biochim Biophys Acta 1022 (1990), 365-372.

[10] S. Kohler, S. Ullrich, U. Richter and U. Schumacher, E-/Pselectins and colon carcinoma metastasis: First in vivo evidence for their crucial role in a clinically relevant model of spontaneous metastasis formation in the lung, Brit J Cancer 102 (2010), 602-609. 
[11] M.C. Manara, G. Bernard, P.-L. Lollini, P. Nanni, M. Zuntini, L. Landuzzi, S. Benini, G. Lattanzi, M. Sciandra, M. Massimo Serra, M.P. Colombo, A. Bernard, P. Picci and K. Scotlandi, CD99 Acts as an Oncosuppressor in Osteosarcoma, Mol Biol Cell 17 (2006), 1910-1921.

[12] R. Matsusue, H. Kubo, S. Hisamori, K. Okoshi, T. Hidekazu, K. Hida, K. Nakano, A. Itami, K. Kawada, S. Nagayama and Y. Sakai, Hepatic stellate cells promote liver metastasis of colon cancer cells by the action of SDF-1/CXCR4 axis, Ann Surg Oncol 16 (2009), 2645-2653.

[13] S. Narayanan, Sialic acid as a tumor marker, Ann Clin Lab Sci 24 (1994), 376-384.

[14] G.N. Naumov, S.M. Wilson, I.C. MacDonald, E.E. Schmidt, V.L. Morris, A.C. Groom, R.M. Hoffman and A.F. Chambers, Cellular expression of green fluorescent protein, coupled with high-resolution in vivo videomicroscopy, to monitor steps in tumor metastasis, J Cell Sci 112 (1999), 1835-1842.

[15] B.P. Pereira, Y. Zhou, A. Gupta, D. Leong, K.Z. Aung, L. Ling, R.W.H. Pho, M. Galindo, M. Salto-Tellez, G.S. Stein, S.M. Cool, A.J. Van Wijnen and S.S. Nathan, Runx2, p53, and pRB status as diagnostic parameters for deregulation of osteoblast growth and differentiation in a new pre-chemotherapeutic osteosarcoma cell line (OS1), J Cell Physiol 221 (2009), $778-788$.

[16] L. Pusztai, Current status of prognostic profiling in breast cancer, Oncologist 13 (2008), 350-360.

[17] A. Rajput, I.D. San Martin, R. Rose, A. Beko, C. LeVea, E. Sharratt, R. Mazurchuk, R.M. Hoffman, M.G. Brattain and J. Wang, Characterization of HCT116 human colon cancer cells in an orthotopic model, World J Surg Res 147 (2008), 276-281.

[18] R.L. Rich and D.G. Myszka, Higher-throughput, label-free, real time molecular interaction analysis, Anal Biochem 361 (2007), 1-6.

[19] B.D. Robinson, G.L. Sica, Y.-F. Liu, T.E. Rohan, F.B. Gertler, J.S. Condeelis and J.G. Jones, Tumor microenvironment of metastasis in human breast carcinoma: A potential prognostic marker linked to hematogenous dissemination, Clin Cancer Res 15 (2009), 2433-2441.

[20] J. Schmidt, K. Liebscher, N. Merten, M. Grundmann, M. Mielenz, H. Sauerwein, E. Christiansen, M.E. Due-Hansen,
T. Ulven, S. Ullrich, J. Gomeza, C. Drewke and E. Kostenis, Conjugated linoleic acids mediate insulin release through islet G protein-coupled receptor FFA1/GPR40, J Biol Chem 286 (2011), 11890-11894.

[21] J. Sleeman and P.S. Steeg, Cancer metastasis as a therapeutic target, Eur J Cancer 46 (2010), 1177-1180.

[22] M.B. Sporn, The war on cancer, Lancet 347 (1996), 1377-1381.

[23] N. Tani, S. Higashiyama, N. Kawaguchi, J. Madarame, I. Ota, Y. Ito, Y. Ohoka, S. Shiosaka, Y. Takada and N. Matsuura, Expression level of integrin a5 on tumour cells affects the rate of metastasis to the kidney, Brit J Cancer 88 (2003), 327-333.

[24] E.W. Thompson, N. Brunner, J. Torri, M.D. Johnson, V. Boulay, A. Wright, M.E. Lippman, P.S. Steeg and R. Clarke, The invasive and metastatic properties of hormone-independent but hormone-responsive variants of MCF-7 human breast cancer cells, Clin Exp Metastasis 11 (1993), 15-26.

[25] M. Toyoshima, N. Tanaka, J. Aoki, Y. Tanaka, K. Murata, M. Kyuuma, H. Kobayashi, N. Ishii, N. Yaegashi and K. Sugamura, Inhibition of tumor growth and metastasis by depletion of vesicular sorting protein Hrs: Its regulatory role on E-cadherin and b-catenin, Cancer Res 67 (2007), 5162-5171.

[26] N. Turner, M.B. Lambros, H.M. Horlings, A. Pearson, R. Sharpe, R. Natrajan, F.C. Geyer, M. van Kouwenhove, B. Kreike, A. Mackay, A. Ashworth, M.J. van de Vijver and J.S. Reis-Filho, Integrative molecular profiling of triple negative breast cancers identifies amplicon drivers and potential therapeutic targets, Oncogene 29 (2010), 2013-2023.

[27] E. Wang, A. Lenferink and M.O. Connor-McCourt, Cancer systems biology: Exploring cancer-associated genes on cellular networks, Cell Mol Life Sci 64 (2007), 1752-1762.

[28] R.A. Weinberg, Mechanisms of malignant progression, Carcinogenesis 29 (2008), 1092-1095.

[29] W.-T. Xu, Z.-Y. Bian, Q.-M. Fan, G. Li and T-T. Tang, Human mesenchymal stem cells (hMSCs) target osteosarcoma and promote its growth and pulmonary metastasis, Cancer Lett $\mathbf{2 8 1}$ (2009), 32-41. 


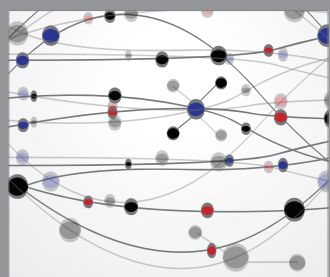

The Scientific World Journal
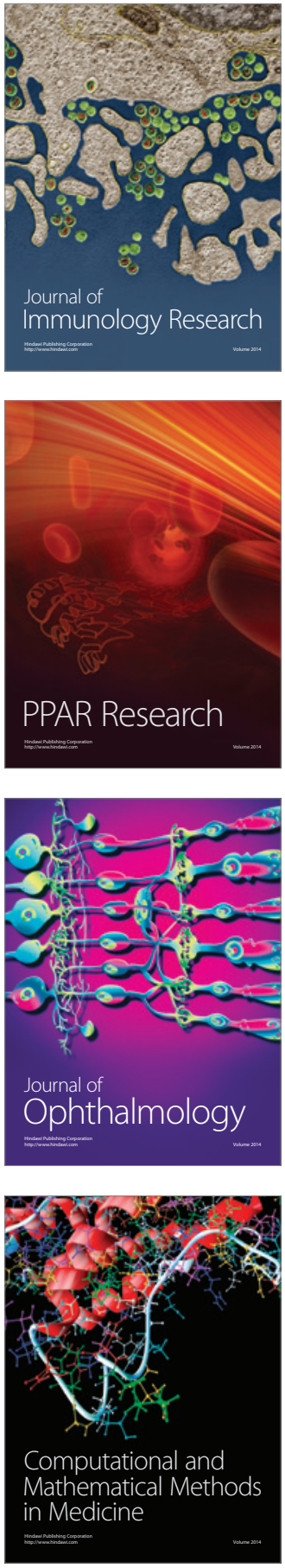

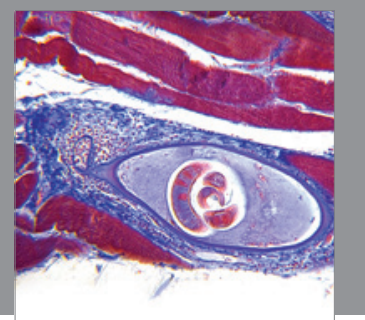

Gastroenterology

Research and Practice
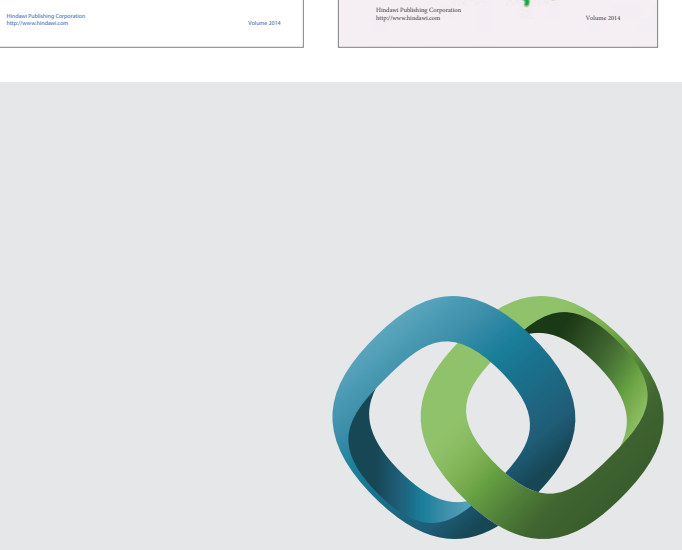

\section{Hindawi}

Submit your manuscripts at

http://www.hindawi.com
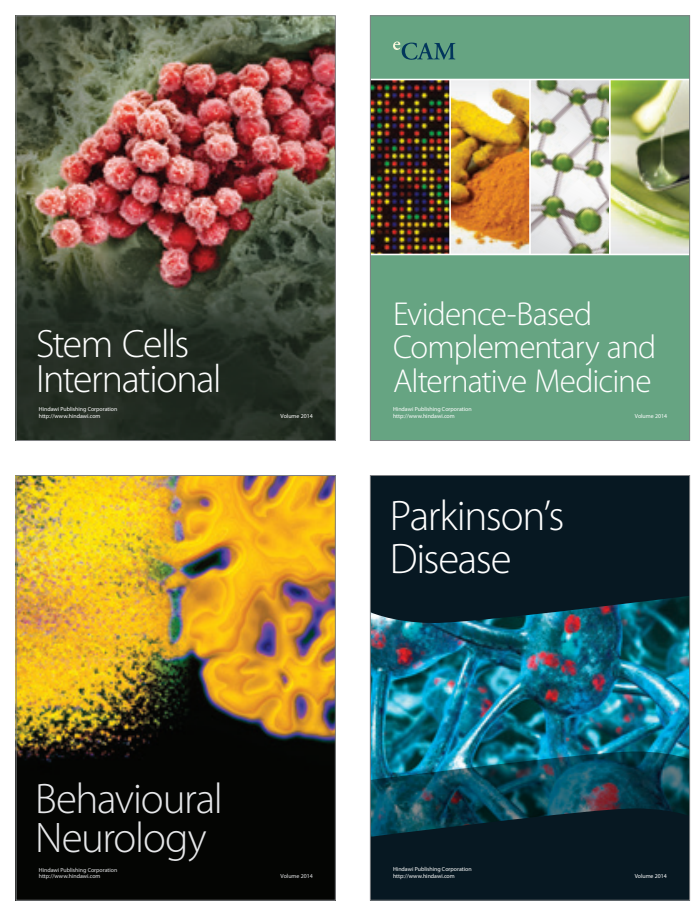

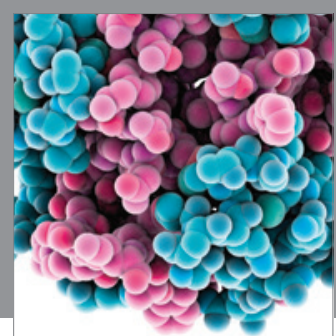

Journal of
Diabetes Research

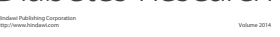

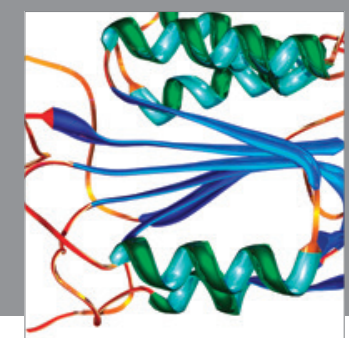

Disease Markers
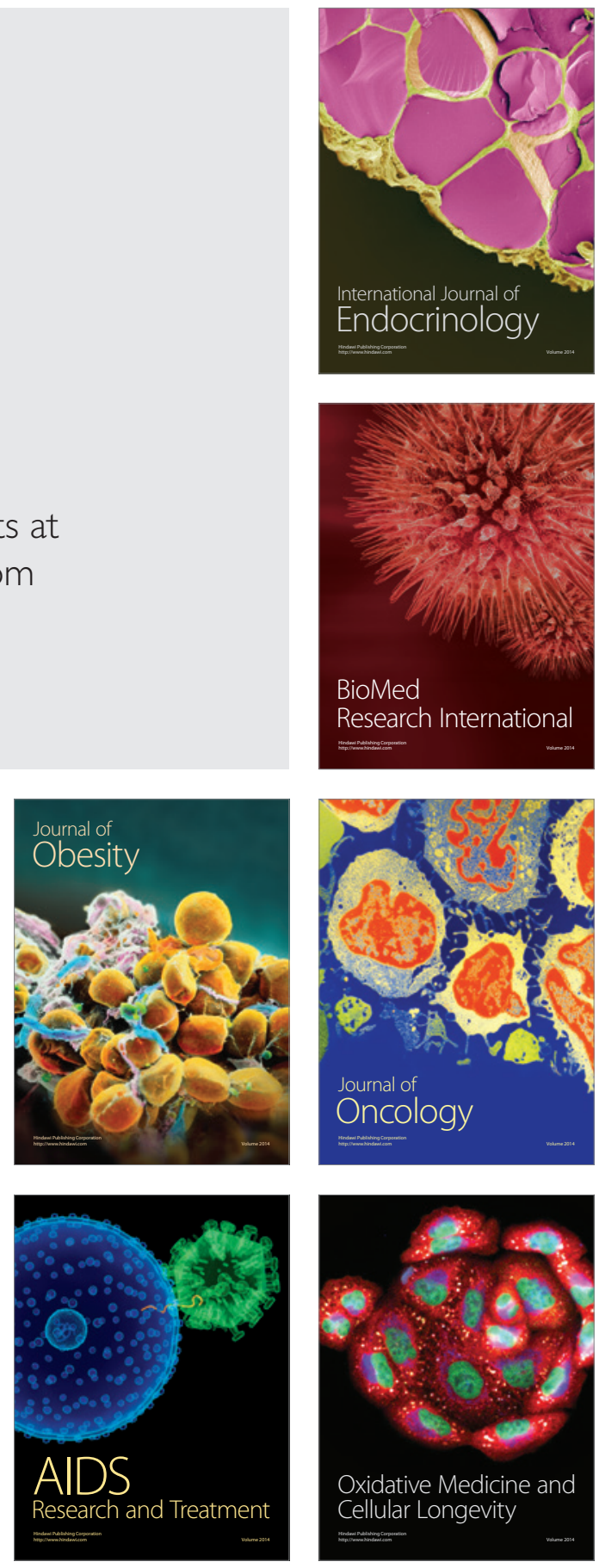